\title{
DISPERSION COMPENSATION USING ELECTRONIC METHODS
}

\author{
Augustin Vyukusenge, \\ University of Bujumbura, Bujumbura, Burundi, \\ vyukusengeaugustin@yahoo.fr
}

DOI: 10.36724/2664-066X-202I-7-I-33-38

\begin{abstract}
System distortions due to chromatic dispersion (CD), polarization mode dispersion (PMD), laser phase noise and fiber nonlinearities have a significant impact on the performance of high-speed fiber optic networks [1]. In connection with the need to improve the quality of information transmission in high-speed fiber-optic communication systems, coherent detection with a digital signal processing unit is of particular interest. The main goal of the DSP block is to reduce the impact of linear and nonlinear effects that degrade the quality of information transfer. In this direction, the use of adaptive filters with adaptation algorithms for filter coefficients plays an important role. The Constant Modulus Algorithm (CMA) and the Least Mean Square (LMS) method used to compensate for dispersion distortions are presented. The load on transport networks based on fiber-optic transmission systems is increasing at an accelerating rate. This paper discusses the possibility and limitations of increasing the throughput of fiber-optic transmission systems by reducing the distance between carriers. A comparison is made between fixed and flexible grids in terms of the spectral bandwidth efficiency. It is concluded that the use of flexible mesh technology is promising when switching to channel speeds above $100 \mathrm{Gbit} / \mathrm{s}$.
\end{abstract}

KEYWORDS: Equalizers, weights, digital signal processing, digital filtering algorithm, Bandwidth, fixed mesh, flexible mesh, number of channels, spectral efficiency, fiber optic transmission system. 


\section{Introduction}

Due to the high spectral transmission efficiency and robust resistance to fiber nonlinearities, coherent optical detection using modern modulation formats and digital signal processing (DSP) has become one of the most promising solutions for the next generation of high-speed fiber-optic transmission systems [2]. Since both amplitude and phase information from the received signal can be extracted using a coherent optical receiver, distortions caused by the above influences can be effectively compensated or reduced using powerful DSP algorithms. $\mathrm{HD}$ and PMD can be well compensated with digital filters in the time and frequency domains [3].

Digital filters have become the most promising alternative approaches to dispersion compensating fibers. These implementations result in significant reductions in complexity and cost as well as improved fiber nonlinearity immunity for high performance fiber optic transmission systems.Filters used to compensate for dispersion can have both constant parameters and adaptive [1]. In the case of using digital equalizers, implemented on the basis of constant parameters for the implementation of static compensation of intersymbol interference, it is necessary to have a priori knowledge of the signal and interference in the transmission line [5].

However, in switched fiber optic networks, the signal path between two end nodes can change over time according to different network conditions, where the transmission distance and accumulated dispersion in the light path cannot be predicted in advance. Therefore, it is advisable to consider adaptive dispersion compensation in such optical transmission networks.

The main feature of an adaptive system is the presence or absence of an exemplary or reference signal. If it is available, the adaptation process is called supervised learning, and the adaptive filter seeks to make its input signal as close as possible to the exemplary signal. If there is no exemplary signal, then the adaptation is called blind, or unsupervised learning, which is a more complex computational problem.

\section{Electronic dispersion compensation techniques}

Digital signal processing using both recovery algorithms and the development of a component base for a digital-to-analog converter makes it possible to eliminate linear optical interference, such as HD, PMD, and phase shift [6]. Adaptive filters play an important role in the implementation of digital compensation.

An adaptive filter (AF) is a system whose parameters are adapted (adjusted) to a signal with a previously undefined statistical model during its processing. Among adaptive filters, linear adaptive feedback filters implemented on the basis of FIR filters are most widely used due to their ease of use.

AF input receives two signals simultaneously:

input signal $\mathrm{x}(\mathrm{k})$ is unknown in advance; desired signal d (k)

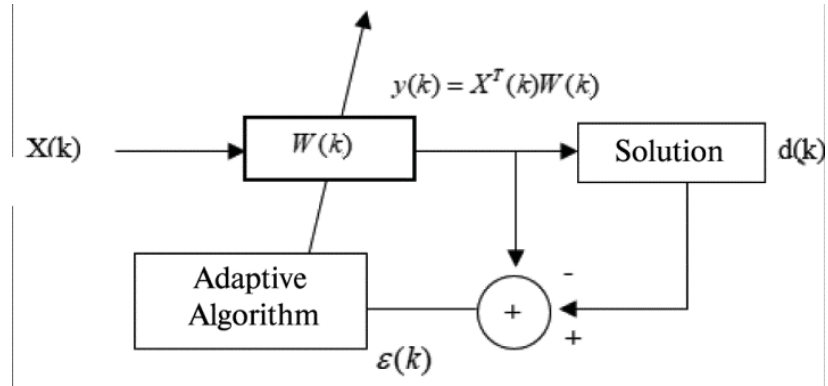

Figure 1. Adaptive equalizer Configuring

The performance of an FIR filter can be expressed as a vector of values known as tap weights. It is these weights that determine the performance of the filter. These values are expressed in vector column form as, $\mathrm{W}(\mathrm{k})=\left[\mathrm{w}_{0}(\mathrm{k}), \mathrm{w}_{1}(\mathrm{k}), \ldots, \mathrm{w}_{\mathrm{N}-1}(\mathrm{k})\right]^{\mathrm{T}}$.

This vector represents the impulse response of the filter of $\operatorname{order} N$. The output from the FIR filter is defined [4]:

$$
y(k)=X^{T}(k) W(k)=\sum_{i=0}^{n} w_{i}(k) x(k-i)
$$

where $X(k)=[x(k), x(k-1), \ldots, x(k-n+1)]^{T}$ column vector of complex filter input.

In theory, given a sufficient number of taps, an adaptive filter can compensate for any kind of linear distortion by adjusting the coefficients of the FIR filter [1-3].

With adaptive compensation, the weights are updated at each time step with the following ratio:

$$
\vec{w}(k+1)=\vec{w}(k)+\mu \varepsilon(k) x(k)
$$

where $\varepsilon(k)$ - signal recovery error at the $k$ step, $\mu-$ step size. This is a convergence parameter that is associated with filters to update the weights. Some difficulty arises when analyzing its behavior, because the higher its value, the faster the convergence, but the accuracy becomes low. On the other hand, a low value gives high accuracy, but slows down convergence.

It is necessary to find such filter coefficients $\vec{w}(k)$, which ensure the maximum proximity of the filter output signal to the reference, that is, minimize the error $\varepsilon(k)$ [3].

Various adaptive algorithms are used to control the equalizer coefficients. Constant Modulus Algorithm (CMA: Constant Modulus Algorithm) and Least Mean Square (LMS: Least Mean Square) are some of the most widely used algorithms used to compensate or reduce the effects of variances in the electrical domain. Both methods rely on the implementation of adaptive filters whose coefficients are updated to minimize RMS error due to intersymbol interference and noise. 


\section{Least mean square method (LMS)}

Purpose of least mean square method is to calculate the difference between reference signal and output of adaptive filter, $\varepsilon(k)$, which is called an error signal and is fed back to the adaptive filter, and its coefficients are changed algorithmically to minimize a function of this difference, known as a cost function [5].

$$
\varepsilon(k)=d(k)-|y(k)|^{2}
$$

If the output of adaptive filter is error-free, then the output of filter converges to desired signal. Larger the error at filter output, greater the deviation between filter output signal and desired signal.

Figure 2 shows the result of dispersion compensation using an equalizer based on LMS algorithm. Dispersion distortions were compensated, but this method converges rather slowly and has increased error variance, as shown by convergence plot.
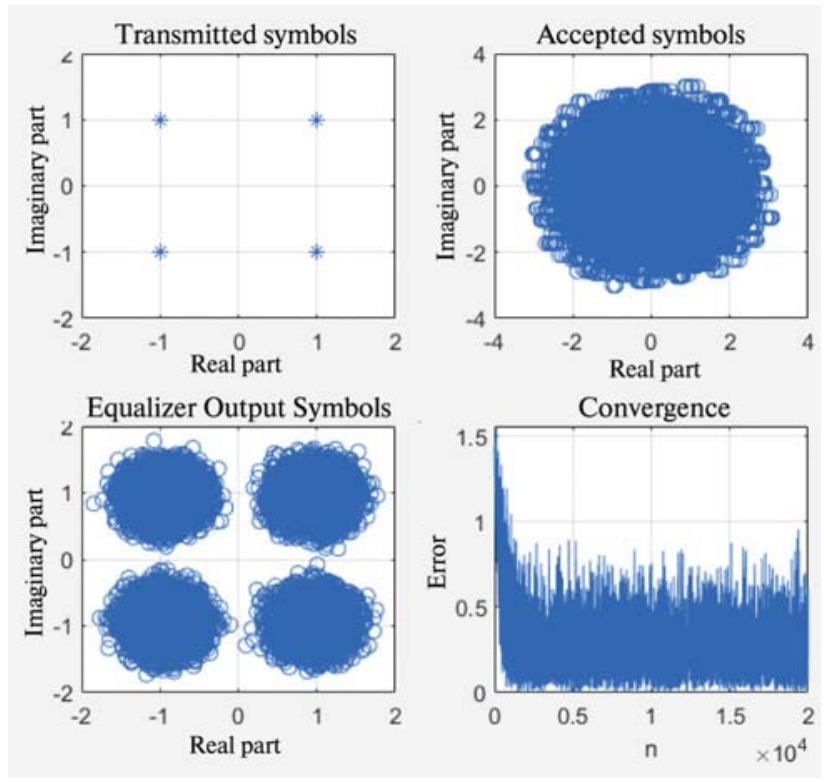

Figure 2. Distortion compensation based on LMS algorithm

\section{Constant modulus algorithm (CMA)}

The use of a constant modulus for calculating the error is based on the fact that the PSK signal without distortion (PSK) has a constant power level. Therefore, the goal is to minimize power level fluctuations by comparison with a constant modulus [5].

$$
\varepsilon(k)=1-|y(k)|^{2}
$$
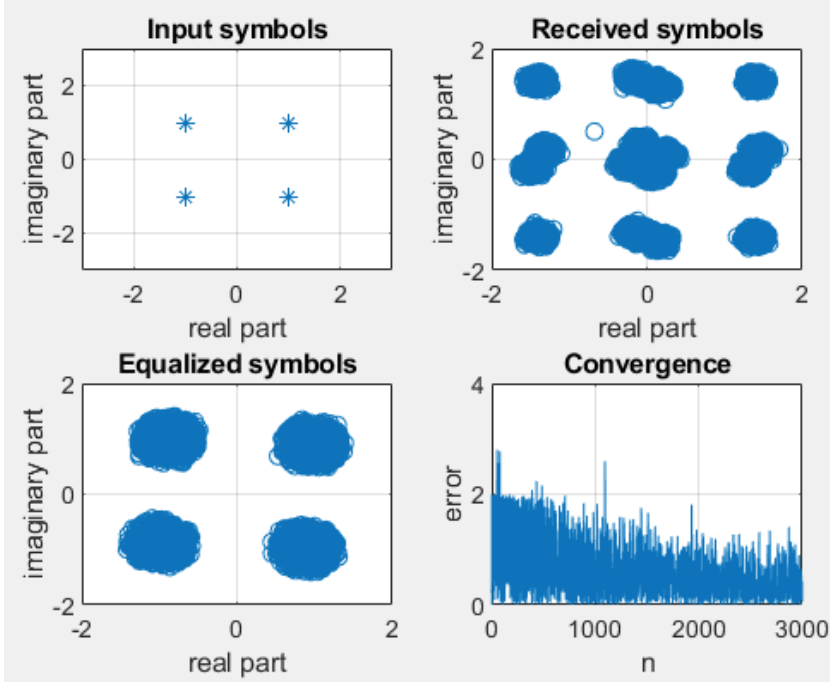

Figure 3. Distortion compensation based on CMA algorithm

It follows from Figure 3 that CMA algorithm is also more efficient for large phase noise, since the performance of LMS algorithm will be significantly degraded due to the large phase noise.

Each of these algorithms has its own advantages and disadvantages. Equalizers based on CMA are difficult to apply directly to M-QAM modulation formats, the envelope of which has a multi-level structure [4]. Equalizers based on LMS, in turn, are sensitive to fluctuations in the carrier phase, which will prevent convergence of the filter coefficients. Therefore, the removal of phase and frequency errors before or during LMS adaptation is essential.

To adapt the filter coefficients, a combined scheme of CMA and LMS algorithms can be applied. In this case, a CMA-based equalizer is first applied to achieve preliminary convergence, followed by frequency and phase equalization, and finally a less reliable but more accurate LMS algorithm is applied. After the adaptation stage, the equalizer coefficients are fixed and used to equalize the received signal.

\section{INCREASING THE BANDWIDTH \\ OF FIBER OPTIC TRANSMISSION SYSTEMS BY DECREASING THE DISTANCE BETWEEN THE CARRIER}

The current period of development of information and communication technologies (ICT) sector is characterized by widespread introduction of a variety of multimedia services, rapid development of Internet networks, emergence of data processing centers, massive introduction of mobile applications requiring high bandwidth and advanced technologies for high-speed packet transmission. Implementation of these trends necessitates a sharp increase in the throughput of fiberoptic transmission systems (FOTS). 
Obviously, under these conditions, of particular interest is a transmission medium that has a high potential throughput and makes it possible to multiply the amount of transmitted information. Thus, fiber-optic cables act as the main transmission medium of the transport network, on the basis of which a layer of transparent optical channels is formed using the technology of spectral multiplexing (DWDM). The data transfer rate achieved by fiber-optic systems over the past 30 years has increased by more than four orders of magnitude [7].

Traffic forecasting in the construction of optical communication networks is a difficult job, which requires the development of methods to increase the bandwidth of FOTS without significant costs for the modernization of expensive line-cable facilities. In this regard, in order to expand the physical network, it is advisable to make more significant efforts to improve the efficiency of fiber-optic information transmission systems. One of the ways to effectively use the capabilities of optical fiber is to increase the number of channels in a frequency band by reducing the distance between channels, the path along which the developers of the G.692 standard have gone [13].

\section{Applying a fixed grid}

The number of channels, channel spacing, width of each channel and the channel bandwidth are important parameters in the design and construction of a high-speed communication network.

In traditional DWDM systems, optical spectrum in the C-band, consisting of approximately 4.1 THz, is divided into hard spectrum intervals of $50 \mathrm{GHz}$ as defined in ITUT Rec. G.694.1. This forms a "grid of wavelengths, where the center frequencies of adjacent channels have fixed spectral intervals of $50 \mathrm{GHz}$. The frequency grid defined in this recommendation supports a variety of fixed channel spacings from $12.5 \mathrm{GHz}$ to $100 \mathrm{GHz}$ or more (integer multiples of $100 \mathrm{GHz}$ ), as well as a flexible grid [8].

When using DWDM technology, one can try to estimate the limiting value of the equivalent FOTS bandwidth, a parameter defined as the product of the transmission rate in the optical channel $\mathrm{B}_{\mathrm{ch}}$ for the number of channels $N$.

$$
\mathrm{B}_{\max }=\mathrm{B}_{\mathrm{ch}} \times \mathrm{N}
$$

Using the standardized range $(192.10-196.10 \mathrm{THz})$, the total frequency width is $4.1 \mathrm{THz}$. If the channel spacing is $50 \mathrm{GHz}$, a maximum of 81 channels can be accommodated. If the channel spacing is reduced to 25 $\mathrm{GHz}$, this range can accommodate 163 channels, and thus double the bandwidth of the FOTS.

While systems with reduced channel spacing will be able to provide significant FOTS bandwidth, this reduction places more stringent demands on the devices used in the system, which reduces the number of potential equipment manufacturers as well as increases costs. From the transmitter's point of view, wavelength stability becomes very important, since even a small drift can cause serious inter-channel interference [9]. With small values of inter-channel gaps, the influence of the effect of four-wave mixing and cross-phase modulation increases, which begins to limit the maximum range of nonregenerative information transmission due to a decrease in the signal-to-noise ratio. A small inter-channel distance can also limit the ability to transmit information at a high channel rate, since there is an overlap of spectra of adjacent channels (Fig. 4).

Interchannel interference together with intersymbol interference presents itself as serious influencing factors that degrade the quality of signal reception. They generate two-dimensional (2D) interference that must be efficiently processed by digital signal processing at the receiver [10].

In the absence of interchannel distortion, intersymbol distortion can be compensated for by an adaptive equalizer and an FEC decoder. Addressing the combined effects of this interference requires some form of joint processing of spectrally overlapping asynchronous WDM channels [10].
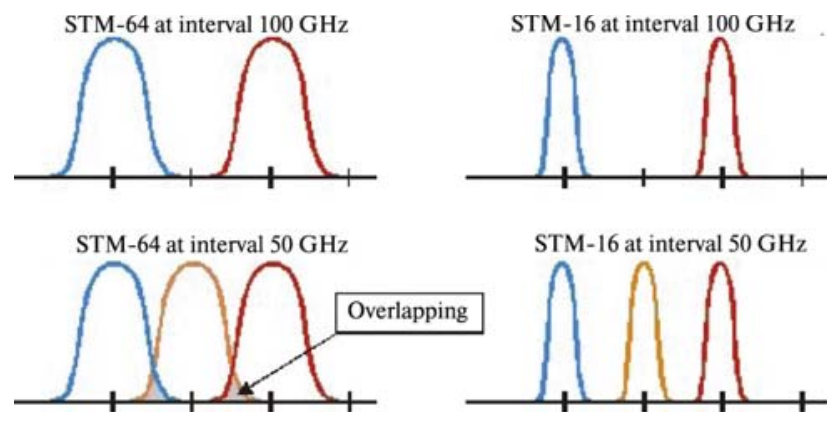

Figure 4. Multiplexing of STM-64 and STM-16 channels at intervals of $100 \mathrm{GHz}$ and $50 \mathrm{GHz}$

The use of advanced modulation formats and photonic techniques allows signals to be transmitted at a channel rate of $100 \mathrm{Gbit} / \mathrm{s}$ in WDM with a fixed grid of $50 \mathrm{GHz}$. However, for higher traffic rates such as $400 \mathrm{Gbps}$ and 1 Tbps, the required bandwidth using standard modulation formats becomes too wide to fit into a $50 \mathrm{GHz}$ grid. To prevent channel-to-channel crosstalk, one option may be to increase the fixed mesh width from $50 \mathrm{GHz}$ to 100 $\mathrm{GHz}$. The disadvantage of using a wider grid is not only that fewer wavelengths will be transmitted, but also that channels with low speed channels will use a grid up to $100 \mathrm{GHz}$ each, which leads to a decrease in the efficiency of spectrum resource use. Another attempt would be to use higher spectral efficiency (SE) modulation formats such as QPSK and QAM. This option leads to a reduction in the transmission range due to the increased requirements of the optical signal-to-noise ratio (OSNR).

\section{Flexible mesh application}

To overcome the aforementioned disadvantages when moving to a transmission rate of more than $100 \mathrm{Gbit} / \mathrm{s}$, Flex Grid technology was proposed, defined in ITU-T Recommendation G.694.1, based on a $12.5 \mathrm{GHz}$ channel spectral separation (Fig. 5). With this technique it becomes possible to flexibly spectral shaping of any channel, including a super channel in a certain frequency range and optical zooming optical network capacity [11]. 


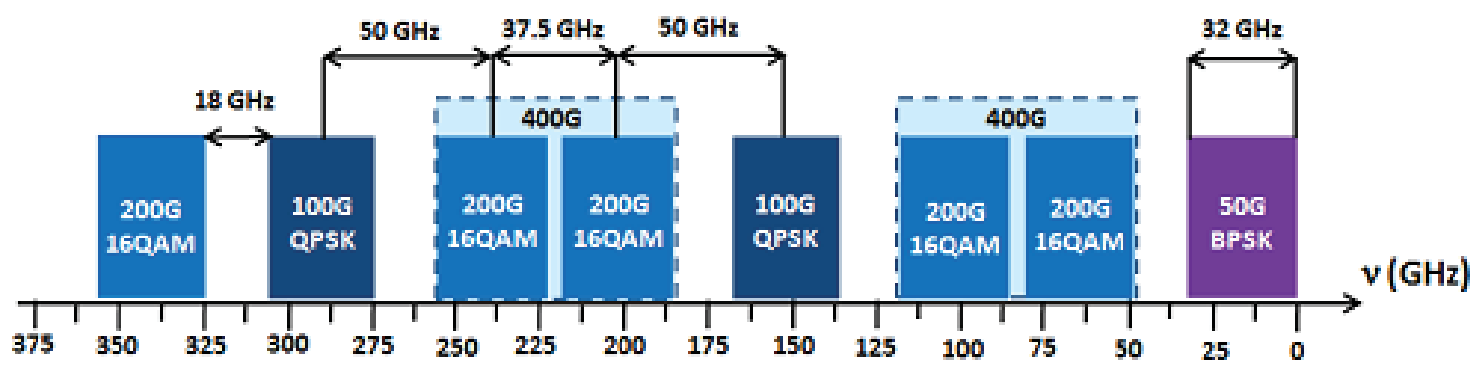

Figure 5. ITU-T G.694.1 flexible frequency grid based on $12.5 \mathrm{GHz}$ grid

Table 1

Comparison of the use of spectral for different modulation formats

\begin{tabular}{|c|l|c|c|c|c|c|}
\hline \multicolumn{2}{|c|}{} & \multicolumn{2}{c|}{ Fixed Grid 50 GHz } & \multicolumn{3}{c|}{ Flexible mesh } \\
\hline $\begin{array}{c}\text { Transfer rate, } \\
{[\mathrm{Gbps}]}\end{array}$ & Modulation format & $\begin{array}{c}\text { Number of } \\
\text { wavelengths }\end{array}$ & $\begin{array}{c}\text { Spectrum } \\
{[\mathrm{GHz}]}\end{array}$ & $\begin{array}{c}\text { Number of } \\
\text { slot }\end{array}$ & $\begin{array}{c}\text { Spectrum } \\
{[\mathrm{GHz}]}\end{array}$ & Capacity gain \\
\hline 10 & $\begin{array}{l}\text { NRZ-O } \\
\text { SE=1бит/с/Гц }\end{array}$ & 1 & 5 & 2 & 25 & $100 \%$ \\
\hline 40 & $\begin{array}{l}\text { DP-QP } \\
\text { SE=4бит/с/Гц }\end{array}$ & 1 & 5 & 2 & 25 & $100 \%$ \\
\hline 100 & $\begin{array}{l}\text { DP-Q } \\
\text { SE=4бит/с/Гц }\end{array}$ & 1 & 5 & 3 & 37.5 & $33.3 \%$ \\
\hline $\begin{array}{l}\text { OF DP-QPSK } \\
\text { SE=4бит/с/Гц }\end{array}$ & 4 & 20 & 10 & 125 & $60 \%$ \\
\hline
\end{tabular}

The possibility of using Flex Grid technology in commercial systems appeared only after the creation and start of mass production of tunable wavelength selective switches (WSS), using LCoS (liquid crystal on silicon) technology [12].

In [11], a theoretical analysis was carried out comparing flexible mesh and standard mesh, taking into account optical channels between 10 Gbps and 400 Gbps. The characteristics of the signals of each grid type (modulation format, spectrum efficiency in bits / symbol and guard band) are given in Table. 1 derived from data from several transmission studies.

As you can see from the table, a $10 \mathrm{Gbps}$ channel using the NRZ-OOK modulation format requires a 25 $\mathrm{GHz}$ slot (two slots) in a flexible mesh, while $50 \mathrm{GHz}$ is used for a fixed mesh. The rest of the channel types (40, 100 and 400 Gbps) use DP-QPSK as the modulation format, reaching a spectral efficiency of 4 bits / symbol. The bandwidth of the spectrum shown in table. 1, takes into account a $7 \mathrm{GHz}$ guard band between optical channels.

The effect of forward error correction is also taken into account by increasing the data rate by $12 \%$. In the case of a fixed mesh, the 400 Gbps link requirements are served by four $100 \mathrm{Gbps}$ links, so 4 carriers are used (total $200 \mathrm{GHz}$ in the case of a $50 \mathrm{GHz}$ grid). The values in the last column indicate that using the DP-QPSK modulation format, the total FOTS bandwidth can theoretically be increased by $33.3 \%$ with 100 Gbps links or $60 \%$ with 400 Gbps links.

The overall increase in FOTS throughput using Flex Grid technology, coupled with the ability to transfer speeds in excess of 100 Gbps over long distances, are consecutive reasons for the development of flexible grid technology. However, from the perspective of the network operator, it is important to know when this increase in FOTS capacity will represent a viable solution for their networks and how this can be implemented. Depending on the existing fixed grid infrastructure, some solutions may not be supported due to the required spectrum bandwidth. For example, transmissions above 100 Gbps using the DP-QPSK or OFDM-DP-QPSK modulation formats are not possible within the fixed 50 $\mathrm{GHz}$ grid. Although there are modulation formats with higher spectral efficiency, such as DP-16-QAM, which can fit into a fixed $50 \mathrm{GHz}$ grid, such formats cause a drop in the maximum transmission range. 


\section{Conclusion}

With high-speed FOTS, it is necessary to improve the quality of information transmission. The use of digital signal processing methods with adaptive filtering algorithms allows you to compensate or reduce the influence of linear and non-linear effects of optical fiber. The algorithms CMA and LMS, which allow to align the received signals by adjusting the coefficients of the FIR filter, are considered. CMA is designed to work with modulation formats that have a constant modulus, that is, a constant amplitude, so the adaptation process is not affected by the presence of a frequency and phase shift. The LMS algorithm is sensitive to phase fluctuations, which requires the removal of phase and frequency errors before or during adaptation based on it.

To achieve high results, a combined algorithm scheme can be considered.

As the ICT industry develops, so does the need to increase bandwidth and maintain bandwidth flexibility. Reducing the distance between channels is one of the ways to increase the bandwidth of the FOTS. However, the effect of co-channel interference (the effect of fourwave mixing and cross-phase modulation), leading to a decrease in the signal-to-noise ratio, must be taken into account. Comparison of flexible mesh and fixed network for different bit rates and modulation formats showed the advantage of flexible mesh in bandwidth efficiency. The use of flexible grid technology (Flex Grid), which allows spectrum management, is the prospect of increasing the efficiency of FOTS operation at channel speeds above 100 Gbit / s.

\section{References}

1. E.V. Pustovalov. Iterative equalizer in the frequency domain with adaptive channel estimation. Information and control systems. 2012. Vol. 57. No. 2. P. 57-66.
2. X.U. Tianhua, G. Jacobsen, J. LI, M. Leeson, S. Popov. Dynamic physical layer equalization in optical communication networks. Optoelectronics and advanced materials - rapid communications. Vol. 12. No. 1-2, May-June 2018.

3. A.B. Sergienko. Adaptive filtering algorithms: Implementation features in MATLAB. Mathematics in applications. 2003. No. 1 (1).

4. K. Kikuchi. Digital coherent optical communication systems: fundamentals and future prospects. IEICE Electronics Express. Vol.8. No.20. P. 1642-1662.

5. O.V. Goryachkin. Methods of blind signal processing and their applications in radio engineering and communication systems. Moscow: Radio and communication, 2003. 230 p.

6. A. Viyyukusenge, A.Yu. Tsym. Coherent receiver with digital signal processing. Communication Bulletin. 2019. No. 8.P. 17-23.

7. E. Desurvire, C. Kazmierski. Science and technology challenges in XXIst century optical communications. C. $R$. Physique 12 (2011). P. 387-416.

8. ITU-T Rec. G.694.1: Spectral grids for WDM applications: DWDM wavelength grid.

9. E. Connolly et al. Cross-Channel Interference Due to Wavelength Drift of Tunable Lasers in DWDM Networks. IEEE Photonics Technology Letters. Vol. 19. No. 8, April 15, 2007.

10. F. Lehmann, P. Ramantanis and Y. Frignac. "Joint Channel Estimation, Interference Mitigation, and Decoding for WDM Coherent Optical Communications," IEEE/OSA Journal of Optical Communications and Networking. Vol. 6. No. 3. 2014. P. 315-325.

11. V.G. Fokin, R.Z. Ibragimov. Optical systems with terabit and petabit transmission rates. tutorial; Siberian State University of Telecommunications and Informatics; department Multichannel telecommunication and optical systems. Novosibirsk, 2016. $162 \mathrm{p}$.

12. A.V. Leonov, O.E. Naniy, M.A. Sleptsov, V.N. Treshchikov Trends in the development of long-distance optical systems. Applied Photonics. 2016. Vol. 3. No. 2.

13. N.N. Slepov. About modern WDM technology and not only. Foton-Express. 2007. No.1. P. 8-16. 